\title{
$\begin{array}{ll}\text { Research Square } & \begin{array}{l}\text { Preprints are preliminary reports that have not undergone peer review. } \\ \text { They should not be considered conclusive, used to inform clinical practice, } \\ \text { or referenced by the media as validated information. }\end{array}\end{array}$
}

\section{Characterization and analysis of some chilling- response WRKY transcription factors in tomato}

\section{Yixuan Wang}

Beijing Forestry University

Kunyang Zhuang ( $\nabla$ zhuangkunyang@sdau.edu.cn )

Shandong Agricultural University

\section{Qingwei Meng}

Shandong Agricultural University

Chen Meng

Marine Agriculture research center

Original Article

Keywords: WRKY, Transcription factors, Chilling stress, Tomato, Protein interactions

Posted Date: September 18th, 2020

DOl: https://doi.org/10.21203/rs.3.rs-75411/v1

License: (c) (1) This work is licensed under a Creative Commons Attribution 4.0 International License. Read Full License 


\section{Abstract}

WRKY transcription factors play various important roles in biotic and abiotic stress. In present study, a total of 81 WRKYs in tomato (Solanum lycopersicum) was identified and their gene structure, phylogeny and sub-location were analyzed. Here, we further analyzed their expression and potential roles under chilling stress. Nevertheless, the predicted chloroplast-located WRKYs are failed to be detected in the chloroplast. Then, 27 SIWRKYS with high chilling-induced mRNA levels (more than 3 fold to the control) are selected from these WRKYS. Promoter analysis showed that some cold stress-related cis-acting elements (CBFs binding site) existed in many promoter regions of these chilling response WRKYS (WRKY2, WRKY50, WRKY59 etc.), implying that these WRKY transcription factors may participate in CBFs mediated pathway under chilling stress. The interaction proteins of WRKYs are essential to affect the DNA binding and transcription regulatory activities of WRKYs, thus controlling its downstream genes expression. Therefore, we predicted and analyzed the protein-protein interactions of those chilling related WRKY transcription factors and then speculated the complex regulatory and functional network of WRKY transcription factors under chilling stress. A better understanding of SIWRKYs would be helpful for providing a theoretical basis for further illustrating the regulatory mechanism of SIWRKYs under chilling stress.

\section{Background}

Chilling stress is a major environmental factor that limits the agricultural productivity and geographical distribution of many plant species (Sanghera et al., 2011). Tomato (Solanum lycopersicum), as a typical warm-season vegetable crops, is very sensitive to chilling stress $\left(0-12{ }^{\circ} \mathrm{C}\right)$ which is common during their growing season. The chilling stress will rigidify the cell membrane, destabilize protein complexes, and impair photosynthesis of tomato (Martin et al., 1981; Ruelland et al., 2009). Under chilling stress, the complex mechanisms have been evolved to improve plant chilling tolerance including multiple signal transduction, transcription factor regulation and protein-protein interactions (Hannah et al., 2015; Chinnusamy et al., 2007; Shi et al., 2018).

As one of the largest transcriptional factors (TFs) families in plants, WRKY TFs with a conserved WRKYGQK motif and a novel zinc-finger-like motif contain more than 70 memebers in Arabidopsis thaliana and S. Iycopersicum (Wu et al., 2005; Madhunita et al., 2014; Chen et al., 2015). Based on its number of WRKY domain and structure of zinc-finger motifs, WRKY proteins are initially classified into three groups (Group I-III) (Eulgem et al., 2000). Following analyses have shown that Group II WRKY proteins are further divided into five subgroups (Ila, Ilb, Ilc, Ild, and Ile) based on the primary amino acid sequence (Rushton et al., 2010; Madhunita et al., 2014). The green algae Chlamydomonas reinhardtii, non-photosynthetic slime mold Dictyostelium discoideum, and unicellular protest Giardi alamblia all contain a single Group I WRKY gene, suggesting that Group I WRKY proteins with two WRKY domains are the ancestors to the other groups of WRKY proteins and the WRKYs origin is before the emergence of photosynthetic eukaryotes (Zhang and Wang, 2005). In Arabidopsis, AtWRKY40, a Group Ila member, can be recruited from nucleus to cytosol to interact with a chloroplast envelope and the cytosolic C-terminus 
spanned magnesium-protoporphyrin IX chelatase $\mathrm{H}$ subunit (ABAR), thus playing a negative role in response to ABA signaling (Shang et al., 2010). This suggests that WRKY family may evolve to be involved in maintaining the connection between the chloroplast and nucleus during the long evolutionary process. However, the roles of WRKYs in chloroplasts are unclear.

WRKY transcription factors function as important components in the regulation of transcriptional reprogramming during plant stress responses (Madhunita et al., 2014). Extensive studies show that many WRKYs are responsive to pathogens or pathogen elicitors. Studies using overexpression lines or mutants of WRKYs have shown that WRKYs can positively or negatively regulate the expressions of hormonerelated or pathogen defence genes (Dong et al., 2003; Lai et al., 2008, 2011). In addition, WRKYs are involved not only in biotic stress responses but also in abiotic stress responses and adaptations. In Arabidopsis, AtWRKY25, AtWRKY26, and AtWRKY33 enhance the tolerance to heat stress through regulating the expression of heat-induced genes, such as AtHSPs (Li et al., 2011). Overexpression of GsWRKY20 reduces the stomatal density and water loss efficiency, thus improving plant drought tolerance (Luo et al., 2013). While GhWRKY68 reduces the resistance to salt and drought stress (Jia et al., 2015).

Transcription factors are significant for cold signaling and tolerance by modulating the expression of related functional genes (Jiang et al., 2020). WRKY family members are also essential to function in cold response. CsWRKY46 of cucumber, OsWRKY71 of rice, and VbWRKY32 of Verbena bonariensis all play roles in improving the cold tolerance (Kim et al., 2016; Ying et al., 2016; Wang et al., 2020). Besides, in mature pollen of Arabidopsis, AtWRKY34 negatively regulated its cold sensitivity through regulating the expression of CBFs (C-repeat binding factors) (Zou et al., 2010). However, the specific molecular mechanism of how WRKYS respond to cold signals and regulate the expression of downstream genes is still very limited.

Tomato is one of the most sensitive crops to chilling stress. Although the function of many individual WRKY genes have been analyzed in tomato (Table 1, most of them function in responses to biotic stress), the chilling responsive pathway mediated by WRKYs remain unclear. Here, we identified 27 chillingresponse WRKY genes by qRT-PCR and RNA-seq assay. Then, the prediction analysis of their functional interaction networks, phosphorylation sites and cis-acting element suggested their potential roles under chilling stress. 
Table 1

The function analysis of some individual WRKY genes in tomato.

\begin{tabular}{|c|c|c|c|}
\hline Gene & Locus & Function & Refs \\
\hline SIWRKY78 & Solyc12g014610 & Plant immunity & Cui et al., 2019 \\
\hline SIWRKY2 & Solyc01g079260 & Plant growth and development & Singh et al., 2020 \\
\hline SIWRKY15 & Solyc02g093050 & $\begin{array}{l}\text { Plant immunity, response to drought and } \\
\text { salt stress }\end{array}$ & Gao et al., 2019 \\
\hline SIWRKY14 & Solyc02g088340 & Plant immunity & $\begin{array}{l}\text { Chinnapandi et al., } \\
2019\end{array}$ \\
\hline SIWRKY58 & Solyc07g066220 & Plant immunity & Hong et al., 2018 \\
\hline SIWRKY63 & Solyc08g067360 & Plant growth and development & $\begin{array}{l}\text { Chinnapandi et al., } \\
2017\end{array}$ \\
\hline SpWRKY14 & Solyc02g088340 & Plant immunity & Cui et al., 2017 \\
\hline SIWRKY9 & Solyc02g032950 & Fruit ripening & Wang et al., 2017 \\
\hline SIWRKY54 & Solyc07g051840 & & \\
\hline SIWRKY5 & Solyc01g095100 & & \\
\hline SIWRKY74 & Solyc10g011910 & & \\
\hline SIWRKY49 & Solyc06g066370 & & \\
\hline SIWRKY68 & Solyc09g014990 & & \\
\hline SIWRKY60 & Solyc08g008280 & & \\
\hline SIWRKY66 & Solyc08g082110 & & \\
\hline SIWRKY69 & Solyc09g015770 & Response to salt stress & Hichri et al., 2017 \\
\hline SIWRKY78 & Solyc12g014610 & Plant immunity & Li et al., 2015 \\
\hline SIWRKY49 & Solyc06g066370 & Plant immunity & Liu et al., 2014 \\
\hline SIWRKY57 & Solyc07go65260 & Response to salt and drought stress & Li et al., 2012 \\
\hline SIWRKY21 & Solyc03g095770 & Plant immunity & Atamian et al., 2012 \\
\hline \multirow[t]{2}{*}{ SIWRKY72 } & SGNU150340 & \multirow[t]{2}{*}{ Plant immunity } & \multirow{2}{*}{$\begin{array}{l}\text { Bhattarai et al., } \\
2010\end{array}$} \\
\hline & SGNU151380 & & \\
\hline SIWRKY68 & Solyc09g014990 & Plant immunity & $\begin{array}{l}\text { Hofmann et al., } \\
2008\end{array}$ \\
\hline SIWRKY49 & Solyc06g066370 & \multirow[t]{2}{*}{ Plant immunity, response to heat stress } & \multirow[t]{2}{*}{ Zhou et al., 2015} \\
\hline SIWRKY68 & Solyc09g014990 & & \\
\hline
\end{tabular}




\section{Materials And Methods \\ Data collection of SIWRKYs}

The DNA, protein and promoter sequences of 81 SIWRKYS were obtained from PlantTFDB (http://planttfdb.cbi.pku.edu.cn/) and Phytozome (https://phytozome.jgi.doe.gov/pz/portal.html), respectively.

\section{Gene structure analysis, phylogenetic tree and motif composition of SIWRKYs}

The gene structures of SIWRKYs were determined by comparing their genomic sequences with predicted coding sequences using the Gene Structure Display Server (http://gsds.cbi.pku.edu.cn/). The conserved motifs analysis of SIWRKY proteins were done by MEME (http://meme-suite.org/tools/meme). In addition, the phylogenetic tree of SIWRKYs was constructed using MEGA7 (Kumar et al., 2016).

\section{The sub-location, post-translational modification and protein interaction analysis of SIWRKYs}

The sub-locations of all SIWRKYs were predicted by LOCALIZER (http://localizer.csiro.au/) and TargetP 1.1 Server (http://www.cbs.dtu.dk/services/TargetP-1.1/index.php). To confirm the subcellular localization of SIWRKY14, SIWRKY33, SIWRKY49, SIWRKY68, SIWRKY78, the full-length sequence of them without the termination codon were subcloned into a 35S-driven pPZP211-green fluorescent protein (GFP) vector using BamH and Sal, Kpnl and Sal, BamH and Sal, Kpnl and Sal, Kpnl and Sall, respectively. The constructed SIWRKYs-GFP vector were then transformed into Agrobacterium GV3101 cells by the freeze-thaw method. The tobacco transient expression method and the observation of fluorescence were carried out following the methods described by Zhuang et al. (2020). The posttranslational modification of 27 chilling response SIWRKY proteins was conducted by P3DB (http://www.p3db.org/). The construction of 27 chilling response SIWRKYs interactions network was based on STRING (https://string-db.org/cgi/network.pl).

\section{Plant growth conditions, chilling treatment and qRT-PCR analysis}

The tomato plants (Solanum lycopersicum, Micro-TOM) were used in this work. Sterilized and incubated method of seeds and the culture conditions of young seedlings were described as Zhuang et al., (2019). Seedlings with five fully developed leaves were used for the chilling treatment $\left(4^{\circ} \mathrm{C}\right)$ in the illuminated incubation chamber (E-41L2, Percival) with a PFD of approximately $400 \mu \mathrm{mol} \mathrm{m} \mathrm{m}^{-2} \mathrm{~s}^{-1}$ and relative humidity of $60-70 \%$ for $12 \mathrm{~h}$. The functional leaves of the treated plants were harvested and frozen in liquid nitrogen. The methods of total RNA extraction, reverse transcription, and qRT-PCR were all performed as described by Zhuang et al., (2019). This assay was performed with three biological replicates. All primers used in this study are listed in Supporting Information Table S3. 


\section{Results}

\section{The phylogenetic analysis, protein and gene structure analysis of WRKY family members of tomato}

The DNA and protein sequences of 81 SIWRKYs were obtained from PlantTFDB (http://planttfdb.cbi.pku.edu.cn/). The 81 transcripts were named from SIWRKY1 to SIWRKY81 based on their order on the tomato chromosomes. Based on these, phylogenetic tree, conserved protein domains and gene structure analysis were shown in Fig. 1. This analysis showed that SIWRKYs with two WRKY domains (motif1 and motif5) were clustered together except WRKY32 and WRKY8 whose motif5 is not very conservative (Fig. 1). WRKY proteins with two WRKY domains are the ancestors to the WRKY proteins with one WRKY domain (Eulgem et al., 2000; Zhang and Wang, 2005). SIWRKY32 and SIWRKY8 may have specific roles in terms of evolution. In addtion, according to gene structure analysis of the SIWRKYS, the SIWRKY members with motif 4 and motif 10 have at least two introns besides SIWRKY46 and SIWRKY10, but most of the other members have only two introns (Fig. 1).

\section{The subcellular localization analysis of SIWRKYs}

The subcellular localization of each SIWRKY was predicted by LOCALIZER and TargetP 1.1 Server. Previous studies showed that most WRKY family members were targeted to nucleus and cytoplasm (Ruelland et al., 2009; Bakshi et al., 2014). Chloroplast is one of the most sensitive sites to chilling stress in plant cells. Here, we want to identify whether there is some chloroplast-located SIWRKYs. Five SIWRKYS (SIWRKY14, SIWRKY33, SIWRKY49, SIWRKY68, SIWRKY78) were predicted to target in the chloroplast (Fig. 2Table). However, the transient expression assay in Nicotiana benthamiana confirmed that SIWRKY14, SIWRKY33, SIWRKY68, SIWRKY78 were all located to the nucleus (Fig. 2A) and we failed to detect the subcellular localization of SIWRKY49.

The up-regulation SIWRKYs under chilling treatment

In tomato, SIWRKYs were found to play roles in heat, drought, salt and biotic stress (Table 1). While the specific functions of them under chilling stress were unclear. 27 up-regulation SIWRKYS (SIWRKY1, SIWRKY2, SIWRKY6, SIWRKY8, SIWRKY11, SIWRKY15, SIWRKY21, SIWRKY23, SIWRKY24, SIWRKY27, SIWRKY29, SIWRKY35, SIWRKY43, SIWRKY45, SIWRKY49, SIWRKY50, SIWRKY56, SIWRKY59, SIWRKY60, SIWRKY61, SIWRKY62, SIWRKY63, SIWRKY64, SIWRKY69, SIWRKY74, SIWRKY79, SIWRKY80) were selected by RNA-seq analysis (Zhuang et al., 2019) and qRT-PCR under $0 \mathrm{~h}, 1.5 \mathrm{~h}, 3 \mathrm{~h}, 6 \mathrm{~h}, 9 \mathrm{~h}$ and $12 \mathrm{~h}$ chilling treatment. All these genes were up-regulated over 3-fold at least in one time site under chilling stress. And the greatest expression increase (nearly 186.54 fold compared with the expression of $0 \mathrm{~h}$ ) was found at $6 \mathrm{~h}$ of SIWRKY43 under chilling treatment (Fig. 2). Moreover, the expression of most of the chilling response SIWRKY genes were peaked at $9 \mathrm{~h}$ under chilling stress except SIWRKY59 and SIWRKY 60 at $3 \mathrm{~h}, S I W R K Y 43$ at $6 \mathrm{~h}$ and SIWRKY49 at $12 \mathrm{~h}$ (Fig. 2). The results suggest that these chilling response SIWRKY genes may participate in different pathway under chilling stress. 
Analysis of putative relationship between these chilling response SIWRKYs and CBFs

In Arabidopsis, C-repeat/DREB binding factors (CBFs) as key transcription factors that function in cold stress (Shi et al., 2018). CBF proteins recognize the CRT/DRE cis-acting element (for example, CCGAC). In addition, WRKY gene have CRT/DRE cis-acting element. Thus, we speculate WRKY gene function as cold response by CBF combining with CRT/DRE cis-acting element of WRKY gene. Most of the promoters of 27 SIWRKYS contain these CBFs binding elements, while SIWRKY6, SIWRKY11, SIWRKY15, SIWRKY27, SIWRKY29, SIWRKY56, SIWRKY60, SIWRKY63, SIWRKY74 do not contain those elements (Table. 2). By contrast, we further analyzed whether the promoter regions of SICBFs contained WRKY bind element (TTGACC/T, Ciolkowski et al., 2008). The analysis showed that all the three SICBFs promoter regions process the WRKY binding region suggesting that some SIWRKYs, especially these WRKYs without the CBFs binding elements as described above, were the pontensial upstream regulator of SICBFS (data was not shown).

\section{Interaction network of these chilling response SIWRKY proteins}

Exploring the interaction proteins of chilling response SIWRKYs is important to understand their regulatory function mechanism under chilling stress. Therefore, we constructed an SIWRKY proteins interaction network based on the data of Arabidopsis homologous proteins using STRING 11.0 to systematically analyze the interaction proteins of these chilling response SIWRKYs (Fig. 4). Chi et al., (2013) summarized that WRKY-WRKY, WRKY-VQ and WRKY-MAPK interactions were the most common interaction relationship of WRKY. Their corresponding genes in Arabidopsis were shown in Table. S1. As shown in Fig. 4, SIWRKY21 seems a central factor to interact with a large number of other chilling response WRKYs including SIWRKY6, SIWRKY24, SIWRKY43, SIWRKY49, SIWRKY60 and SIWRKY61, which may play the most important roles under chilling stress. Besides, MPK3, MPK2 and MPKA1 may function as a key complex to interact with a large number of chilling response SIWRKYs directly (SIWRKY21, SIWRKY24, SIWRKY49, SIWRKY50, SIWRKY64, SIWRKY74 and SIWRKY79) and then phosphorylated them to affect their transcription regulatory activities (Fig. 4). The post-translational modification analysis showed that all chilling response WRKYs had phosphorylation sites (Table. S3). As expected, these WRKYs, which interact with MPKs as description above, process at least 8 phosphorylation sites, suggesting their interaction relationship (Table. S3). VQ proteins were found to specifically interact with the WRKY domain of WRKY proteins (Cheng et al., 2012). In this analysis, VQ proteins are also predicted to interact with chilling response SIWRKYs (SIWRKY11, SIWRKY24, SIWRKY49, SIWRKY56, SIWRKY68 and SIWRKY74) (Fig. 4). In addition, many other chilling related transcription factors (like ICE1, ERF, AP2, JAZ1 etc.) were found to interact with chilling response SIWRKYs, which was independent with the interaction as described above (Fig. 4). The interaction network of these chilling response SIWRKYs provides new research ideas for exploring the new chilling related mechanism of tomato in the future.

\section{Conclusion}


Almost all studied WRKY proteins can bind to the core W-box promoter elements of their downstream genes in the nucleus to participate in plant growth, development, and responses to biotic and abiotic stress, respectively (Bakshi et al., 2014). As a nucleus and cytosol dual-located WRKY member, AtWRKY40 could be from nucleus to cytosol to interact with a chloroplast envelope located cytosolic Cterminus spanned magnesium-protoporphyrin IX chelatase H subunit (ABAR) (Shang et al., 2010). This suggests that WRKY family may be involved in maintaining the connection between the chloroplast, cytosol and nucleus. However, the affection of WRKYs to the chloroplast is not directly. In tomato, SIWRKYs were predicted to target to the nucleus, cytosol, chloroplasts and mitochondria (Chen et al., 2015). Our result also showed five candidate chloroplast-located SIWRKYs (Fig. 2). Nevertheless, four of them were confirmed to target to the nucleus (Fig. 2). Only the sub-location of SIWRKY49 remains unclear.

CBF, also known as dehydration responsive element (DRE) binding factor (DREB) proteins, are key factors in plant cold response (Liu et al., 1998; Shi et al., 2018). In rice, WRKY genes expressed highly under cold stress and may be involved in ICE-CBF-COR pathway (Guo et al., 2019). In this study, we mainly identified 27 chilling response SIWRKY genes based on RNA-seq and qRT-PCR analysis (Fig. 3). Promoter analysis of SIWRKYS and SICBFS showed most SIWRKYS may have close regulatory relationship with SICBFS mediated chilling response pathway. The promoter regions of 18 chilling response SIWRKYS (SIWRKY1, SIWRKY2, SIWRKY8, SIWRKY21, SIWRKY23, SIWRKY24, SIWRKY35, SIWRKY43, SIWRKY45, SIWRKY49, SIWRKY50, SIWRKY59, SIWRKY61, SIWRKY62, SIWRKY64, SIWRKY69, SIWRKY79 and SIWRKY80) all contained the CBFs binding element, suggesting that they may be the directly downstream signaling factors under chilling stress (Table 2). The SIWRKY6, SIWRKY11, SIWRKY15, SIWRKY27, SIWRKY29, SIWRKY56, SIWRKY60, SIWRKY63, SIWRKY74, which were left behind, may act as upstream regulatory factors of SICBFs due to the W-box elements in SICBFs promoters. Meanwhile, we can not fully rule out that these chilling response SIWRKYs function in a CBFs independent pathway under chilling stress.

Table 2

The chilling related cis-acting element prediction in the promoter region of the 27 SIWRKYS

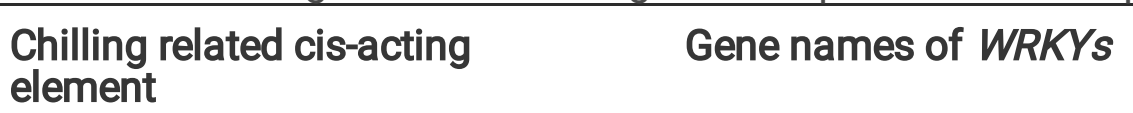

\section{Core Cis-acting element \\ sequence}

RCCGAC DRECRTCOREAT

GTCGAC CRTDREHVCBF2

CCGAC LTRECOREATCOR15

CCGAAA LTRE-1
WRKY2, WRKY21, WRKY24, WRKY35, WRKY43, WRKY50, WRKY59, WRKY69

WRKY2, WRKY23, WRKY43, WRKY50, WRKY59, WRKY62

WRKY1, WRKY2, WRKY8, WRKY21, WRKY23, WRKY24, WRKY35, WRKY43, WRKY49, WRKY50, WRKY59, WRKY61, WRKY69, WRKY79

WRKY1, WRKY2, WRKY8, WRKY21, WRKY23, WRKY24, WRKY35, WRKY43, WRKY49, WRKY50, WRKY59, WRKY61, WRKY69, WRKY79 
The interaction proteins of WRKYs are essential to affect the DNA binding and transcription regulatory activities of WRKYs, thus controlling its downstream genes expression. WRKY-WRKY, WRKY-VQ and WRKY-MAPK interactions are the most common interaction relationship of WRKY (Chi et al., 2013). In Arabidopsis, MPK3, MPK4, and MPK6 could be activated by biotic and abiotic stresses and their functional analyses indicate their critical roles in plant disease resistance and stress tolerance (Pitzschke et al., 2009). AtWRKY33 acts as a downstream component of MPK3/MPK6 cascade in regulation of the pathogen-induced defense responses (Mao et al., 2011). In our analysis, these chilling response SIWRKYs were also predicted to interact with some kinase (MPK3, MPK2 and MPKA1), which may function as a key complex in whole SIWRKYs interaction network under chilling stress in tomato (Fig. 4). In addition, SIWRKY21 seems a central factor due to its interaction with the most chilling response WRKYs. As its homologous gene in Arabidopsis, AtWRKY70 can play a role in plant immunity and response to salicylic acid (SA), reactive oxygen species (ROS) and jasmonic acid (JA) which are also induced by chilling stress (Chen et al., 2017; Lortzing et al., 2018; Zhou et al., 2018; Chae et al., 2020). These suggest that SIWRKY21 may play an essential and complex role in chilling response pathway. Otherwise, SIWRKY23 was predicted to interact with a critical cold-responce factor ICE1 (Induced of CBF expression1, Lee et al., 2005) which showed its potential function under chilling stress.

Although chilling response WRKYs were studied in several species, the mechanism of how WRKYs respond to chilling stress and the specific regulatory mechanism to their downstream genes are remain poorly understood. In this study, we identified 27 obvious chilling-induced SIWRKYS and analyzed their potential roles and interaction network under chilling stress, which provides an important reference for future studies on their biological functions under chilling stress in tomato.

\section{Abbreviations}

WRKYs, WRKY transcription factors; TFs, transcriptional factors; CBFs, C-repeat binding factors; qRT-PCR, quantitative real-time polymerase chain reaction; SA, salicylic acid; ROS, reactive oxygen species; JA, jasmonic acid; ICE1, induced of CBF expression 1

\section{Declarations}

\section{Availability of data and material:}

The data that support the findings of this study are available from the corresponding author upon request.

\section{Competing interests:}

There is no competing interests about this research and all authors agree to publish. 
Not applicable.

\section{Consent for publication:}

Not applicable.

\section{Funding:}

This work was supported by the State Key Basic Research and Development Plan of China (grant number 2015CB150105), the National Natural Science Foundation of China (grant number 31870239, 31870277, 31900208), the Natural Science Foundation of Shandong Province (grant number ZR2018QC001) and Funds of Shangdong "Double Tops" Program.

\section{Author contributions:}

W. M., Y. X. W., C. M. and K. Y. Z. conceived and designed this experiment. K. Y. Z. and C. M. carried out the experiments and analyzed the data. Y. X. W. helped to analyze the data and make the figures. K. Y. Z. and C. M. wrote the manuscript. Q. W. M. and Y. X. W. helped to revise the manuscript. All authors read and approved the manuscript.

\section{Acknowledgements:}

Not applicable.

\section{References}

1. Atamian HS, Eulgem T, Kaloshian I (2012) SIWRKY70 is required for Mi-1-mediated resistance to aphids and nematodes in tomato. Planta 235(2):299-309

2. Chinnapandi B, Bucki P, Miyara SB (2017) SIWRKY45, nematode-responsive tomato WRKY gene, enhances susceptibility to the root knot nematode; M. javanica infection. Plant Signaling Behavior 12(12):e1356530

3. Bhattarai K, Atamian HS, Kaloshian I, Eulgem T (2010) WRKY72-type transcription factors contribute to basal immunity in tomato and Arabidopsis as well as gene-for-gene resistance mediated by the tomato R gene Mi-1. Plant J 63(2):229-240

4. Madhunita B, Ralf O (2014) WRKY transcription factors. Plant Signaling Behavior 9(2):e27700

5. Cao SL, Wang Y, Li XT, Gao F, Feng JC, Zhou YJ (2020) Characterization of the AP2/ERF transcription factor family and expression profiling of DREB subfamily under cold and osmotic stresses in 
Ammopiptanthus nanus. Plants 9(4):455

6. Chae DH, Kim DR, Cheong MS, Lee YB, Kwak YS (2020) Investigating the induced systemic resistance mechanism of 2,4-Diacetylphloroglucinol (DAPG) using DAPG hydrolase-transgenic Arabidopsis. The Plant Pathology Journal 36(3):255-266

7. Chen L, Yang Y, Liu C, Zheng YY, Xu MS, Wu N, Sheng JP, Shen L (2015) Characterization of WRKY transcription factors in Solanum lycopersicum reveals collinearity and their expression patterns under cold treatment. Biochem Biophys Res Commun 464(3):962-968

8. Chen JN, Nolan TM, Ye HX, Zhang MC, Tong HN, Xin PY, Chu JF, Chu CC, Li ZH, Yin YH (2017) Arabidopsis WRKY46, WRKY54, and WRKY70 transcription factors are involved in Brassinosteroidregulated plant growth and drought responses. Plant Cell 29(6):1425-1439

9. Cheng Y, Zhou Y, Yang Y, Chi YJ, Zhou J, Chen JY, Wang F, Fan BF, Shi K, Zhou YH, Yu JQ, Chen ZX (2012) Structural and functional analysis of VQ motif-containing proteins in Arabidopsis as interacting proteins of WRKY transcription factors. Plant Physiol 159(2):810-825

10. Chinnapandi B, Bucki P, Fitoussi N, Kolomiets M, Borrego E, Miyara SB (2019) Tomato SIWRKY3 acts as a positive regulator for resistance against the root-knot nematode Meloidogyne javanica by activating lipids and hormone-mediated defense-signaling pathways. Plant Signaling Behavior 14(6):1601951

11. Chinnusamy V, Zhu JH, Zhu JK (2007) Cold stress regulation of gene expression in plants. Trends Plant Sci 12(10):444-451

12. Cui J, Jiang N, Meng J, Yang GL, Liu WW, Zhou XX, Ma N, Hou XX, Luan YS (2019) LncRNA33732respiratory burst oxidase module associated with WRKY1 in tomato-Phytophthora infestans interactions. Plant J 97(5):933-946

13. Cui J, Xu PS, Meng J, Li JB, Jiang N, Luan YS (2017) Transcriptome signatures of tomato leaf induced by Phytophthora infestans and functional identification of transcription factor SpWRKY3. Theor Appl Genet 131(4):787-800

14. Dong JX, Chen CH, Chen ZX (2003) Expression profiles of the Arabidopsis WRKY gene superfamily during plant defense response. Plant Mol Biol 51(1):21-37

15. Eulgem T, Rushton PJ, Robatzek S, Somssich IE (2000) The WRKY superfamily of plant transcription factors. Trends Plant Sci 5(5):199-206

16. Gao YF, Liu JK, Yang FM, Zhang GY, Wang D, Zhang L, Ou YB, Yao YA (2020) The WRKY transcription factor WRKY8 promotes resistance to pathogen infection and mediates drought and salt stress tolerance in Solanum lycopersicum. Physiol Plant 168(1):98-117

17. Guo H, Wu TK, Li SX, He Q, Yang ZL, Zhang WH, Gan Y, Sun PY, Xiang GL, Zhang HY, Deng HF (2019) The methylation patterns and transcriptional responses to chilling stress at the seedling stage in rice. Int J Mol Sci 20(20):5089

18. Hannah MA, Heyer AG, Hincha DK (2005) A global survey of gene regulation during cold acclimation in Arabidopsis thaliana. PLOS Genetics 1(2):e26 
19. Hichri I, Muhovski Y, Žižková E, Dobrev PI, Gharbi E, Franco-Zorrilla JM, Lopez-Vidriero I, Solano R, Clippe A, Errachid A, Motyka V, Lutts S (2017) The Solanum lycopersicum WRKY3 transcription factor SIWRKY3 is involved in salt stress tolerance in tomato. Front Plant Sci 8:1343

20. Hofmann MG, Sinha AK, Proels RK, Roitsch T (2008) Cloning and characterization of a novel LpWRKY1 transcription factor in tomato. Plant Physiology and Biochemistry, 46(5-6): $533-40$

21. Hong YH, Cui J, Liu Z, Luan YS (2018) SpWRKY6 acts as a positive regulator during tomato resistance to Phytophthora infestans infection. Biochem Biophys Res Commun 506(4):787-792

22. Jia HH, Wang C, Wang F, Liu SH, Li GL, Guo XQ (2015) GhWRKY68 reduces resistance to salt and drought in transgenic Nicotiana benthamiana. PLOS ONE 10(3):e0120646

23. Kim C, Vo KT, Nguyen CD, Jeong D, Lee S, Kumar M, Kim S, Park S, Kim J, JeonJeon J (2016) Functional analysis of a cold-responsive rice WRKY gene, OsWRKY71. Plant Biotechnology Reports 10(1):13-23

24. Lai ZB, Li Y, Wang F, Cheng Y, Fan BF, Yu JQ, Chen ZX (2011) Arabidopsis sigma factor binding proteins are activators of the WRKY33 transcription factor in plant defense. Plant Cell 23(10):38243841

25. Lai ZB, Vinod KM, Zheng ZY, Fan BF, Chen ZX (2008) Roles of Arabidopsis WRKY3 and WRKY4 transcription factors in plant responses to pathogens. BMC Plant Biol 8(1):68-68

26. Li JB, Luan YS, Jin H (2012) The tomato SIWRKY gene plays an important role in the regulation of defense responses in tobacco. Biochem Biophys Res Commun 427(3):671-676

27. Li JB, Luan YS, Liu Z (2015) Overexpression of SpWRKY1 promotes resistance to Phytophthora nicotianae and tolerance to salt and drought stress in transgenic tobacco. Physiol Plant 155(3):248266

28. Li SJ, Fu QT, Chen LG, Huang WD, Yu DQ (2011) Arabidopsis thaliana WRKY25, WRKY26, and WRKY33 coordinate induction of plant thermotolerance. Planta, 233(6): 1237-1252

29. Liu B, Hong YB, Zhang YF, Li XH, Huang L, Zhang HJ, Li DY, Song FM (2014) Tomato WRKY transcriptional factor SIDRW1 is required for disease resistance against Botrytis cinerea and tolerance to oxidative stress. Plant Sci 227(5):145-156

30. Lortzing V, Oberländer J, Lortzing T, Tohge T, Steppuhn A, Kunze R, Hilker M (2018) Insect egg deposition renders plant defense against hatching larvae more effective in a salicylic acid-dependent manner. Plant Cell Environment 42(3):1019-1032

31. Luo X, Bai X, Sun XL, Zhu D, Liu BH, Ji W, Cai H, Cao L, Wu J, Hu MR, Liu X, Tang LL, Zhu YM (2013) Expression of wild soybean WRKY20 in Arabidopsis enhances drought tolerance and regulates ABA signalling. J Exp Bot 64(8):2155-2169

32. Mao GH, Meng XZ, Liu YD, Zheng ZY, Chen ZX, Zhang SQ (2011) Phosphorylation of a WRKY transcription factor by two pathogen-responsive MAPKs drives phytoalexin biosynthesis in Arabidopsis. Plant Cell 23(4):1639-1653

33. Martin B, Ort DR, Boyer JS (1981) Impairment of photosynthesis by chilling-temperatures in tomato. Plant Physiol 68(2):329-334 
34. Pitzschke A, Schikora A, Hirt H (2009) MAPK cascade signalling networks in plant defence. Curr Opin Plant Biol 12(4):421-426

35. Liu Q, Kasuga M, Sakuma Y, Abe H, Miura S, Yamaguchi-Shinozaki K, Shinozaki K (1998) Two transcription factors, DREB1 and DREB2, with an EREBP/AP2 DNA binding domain separate two cellular signal transduction pathways in drought- and low-temperature-responsive gene expression, respectively, in Arabidopsis. Plant Cell 10(8):1391-1406

36. Ruelland E, Vaultier M, Zachowski A, Hurry V, Kader J, Delseny M (2009) Cold signalling and cold acclimation in plants. Advances in Botanical Research 49(8):35-150

37. Rushton PJ, Somssich IE, Ringler P, Shen QJ (2010) WRKY transcription factors. Trends Plant Sci 15(5):247-258

38. Sanghera GS, Wani SH, Hussain W, Singh NB (2011) Engineering cold stress tolerance in crop plants. Curr Genomics 12(1):30-43

39. MEGA7: molecular evolutionary genetics analysis version 7.0 for bigger datasets

Sudhir K, Glen S, Koichiro T (2016) MEGA7: molecular evolutionary genetics analysis version 7.0 for bigger datasets. Mol Biol Evol, 33(7): 1870-1874

40. Shang Y, Liu YL, Cao ZQ, Mei Z, Xin C, Wu Q, Wang FQ, Du XF, Jiang SY, Zhang T, Zhao XF, Sun R, Liu HL, Yu R, Zhang YT D. P (2010) The Mg-chelatase H subunit of Arabidopsis antagonizes a group of WRKY transcription repressors to relieve ABA-responsive genes of inhibition. Plant Cell 22(6):19091935

41. Shi YT, Ding YL, Yang SH (2018) Molecular regulation of CBF signaling in cold acclimation. Trends Plant Sci 23(7):623-637

42. Singh D, Debnath P, Roohi Sane AP, Sane V. A (2020) Expression of the tomato WRKY gene, SIWRKY23, alters root sensitivity to ethylene, auxin and ja and affects aerial architecture in transgenic Arabidopsis. Physiology Molecular Biology of Plants 26(6):1187-1199

43. Wang L, Zhang XL, Wang L, Tian YA, Jia N, Chen SZ, Shi NB, Huang XM, Zhou C, Yu YW, Zhang ZQ, Pang XQ (2017) Regulation of ethylene-responsive SIWRKYs involved in color change during tomato fruit ripening. Sci Rep 7(1):16674

44. Wang MQ, Huang QX, Lin P, Zeng QH, Li Y, Liu QL, Zhang L, Pan YZ, Jiang BB, Zhang F (2020) The Overexpression of a transcription factor gene VbWRKY32 enhances the cold tolerance in Verbena bonariensis. Front Plant Sci 10:1746

45. Wu KL, Guo ZJ, Wang HH, Li J (2005) The WRKY family of transcription factors in Rice and Arabidopsis and their origins. DNA Res 12(1):9-26

46. Zhang Y, Yu HJ, Yang XY, Li Q, Ling J, Wang H, Gu XF, Huang XW, Jiang WJ. CsWRKY46, a WRKY transcription factor from cucumber, confers cold resistance in transgenic-plant by regulating a set of cold-stress responsive genes in an ABA-dependent manner. Plant Physiology and Biochemistry, 108: 478-487

47. Zhang YJ, Wang LJ (2005) The WRKY transcription factor superfamily: its origin in eukaryotes and expansion in plants. BMC Evol Biol 5(1):1-12 
48. Zhou J, Wang J, Zheng ZY, Fan BF, Yu JQ, Chen ZX (2015) Characterization of the promoter and extended C-terminal domain of Arabidopsis WRKY33 and functional analysis of tomato WRKY33 homologues in plant stress responses. J Exp Bot 66(15):4567-4583

49. Zhou M, Lu Y, Bethke G, Harrison BT, Hatsugai N, Katagiri F, Glazebrook J (2018) WRKY70 prevents axenic activation of plant immunity by direct repression of SARD1. New Phytol 217(2):700-712

50. Zhou QY, Tian AG, Zou HF, Xie ZM, Lei G, Huang J, Wang CM, Wang HW, Zhang JS, Chen SY (2008) Soybean wrky-type transcription factor genes, GmWRKY13, GmWRKY21, and GmWRKY54, confer differential tolerance to abiotic stresses in transgenic Arabidopsis plants. Plant Biotechnol $\mathrm{J}$ 6(5):486-503

51. Zhuang KY, Kong FY, Zhang S, Meng C, Yang MM, Liu ZB, Wang Y, Ma NN, Meng QW (2019) Whirly1 enhances the tolerance to chilling stress in tomato via protection of photosystem II and regulation of starch degradation. New Phytol 221(4):1998-2012

52. Zou CS, Jiang WB, Yu DQ (2010) Male gametophyte-specific WRKY34 transcription factor mediates cold sensitivity of mature pollen in Arabidopsis. J Exp Bot 61(14):3901-3914

\section{Figures}



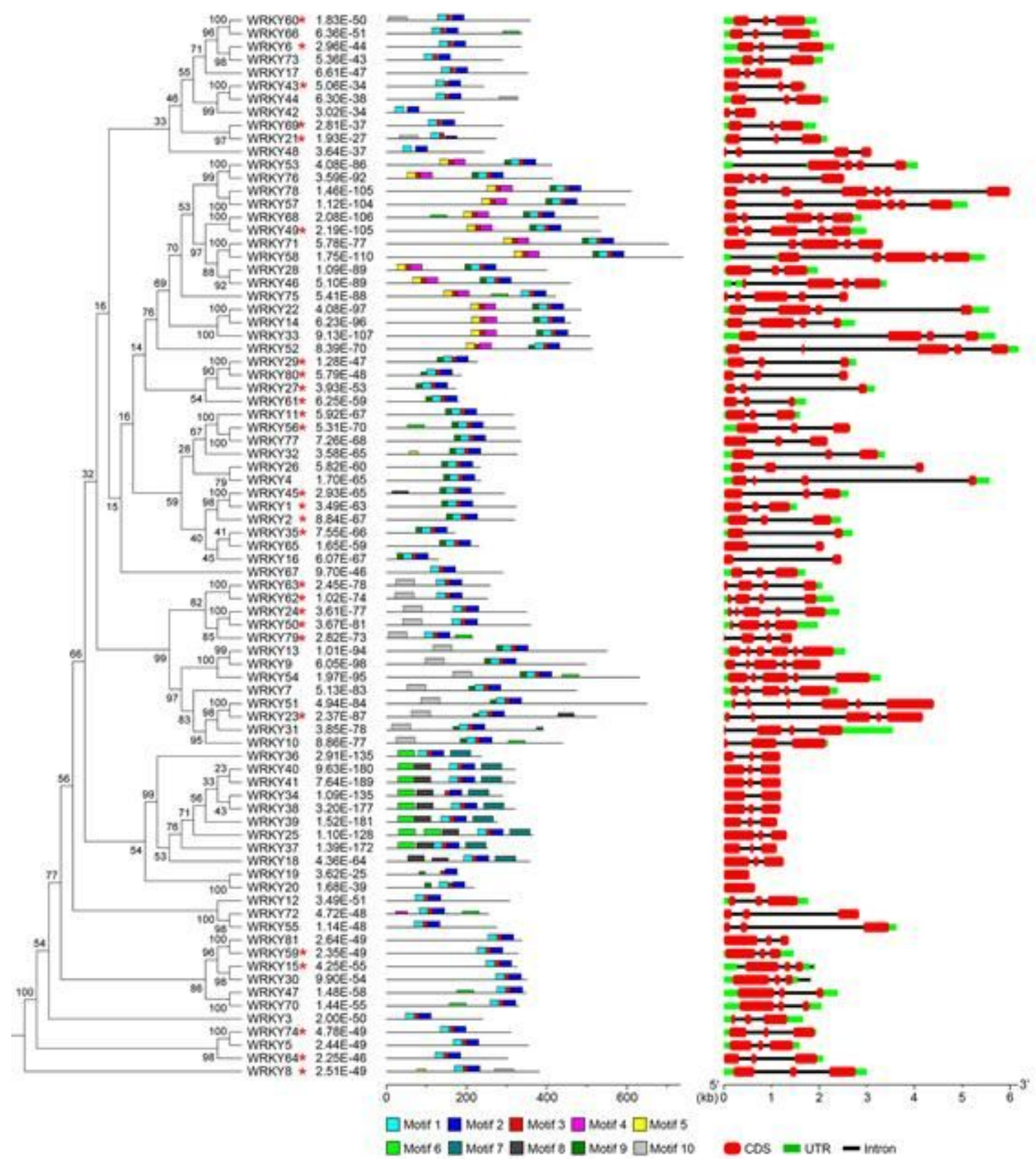

\section{Figure 1}

The phylogenetic analysis, protein and gene structure analysis of SIWRKY family members The phylogenetic analysis shown on the left and the red * indicated the 27 up-regulation SIWRKYs under chilling treatment. The motif composition analysis shown on the middle. WRKY domain was in the motif1 and motif5. Gene structure of SIWRKYs was shown on the right part. 
Table. The prediction and analysis of WRKY subcellular localization.

\begin{tabular}{|c|c|c|c|}
\hline \multirow{2}{*}{ Gene ID } & Gene & \multicolumn{2}{|c|}{ Subcellular location } \\
\cline { 3 - 4 } & name & LOCALIZER & TargetP 1.1 Server \\
\hline Solyc01g079360 & WRKY3 & nucleus & mitochondrion \\
\hline Solyc02g088340 & WRKY14 & nucleus & chloroplast \\
\hline Solyc05g012770 & WRKY33 & nucleus & chloroplast \\
\hline Solyc06g066370 & WRKY49 & chloroplast and nucleus & - (Any other location) \\
\hline Solyc09g014990 & WRKY68 & nucleus & chloroplast \\
\hline Solyc12g014610 & WRKY78 & nucleus & chloroplast \\
\hline
\end{tabular}
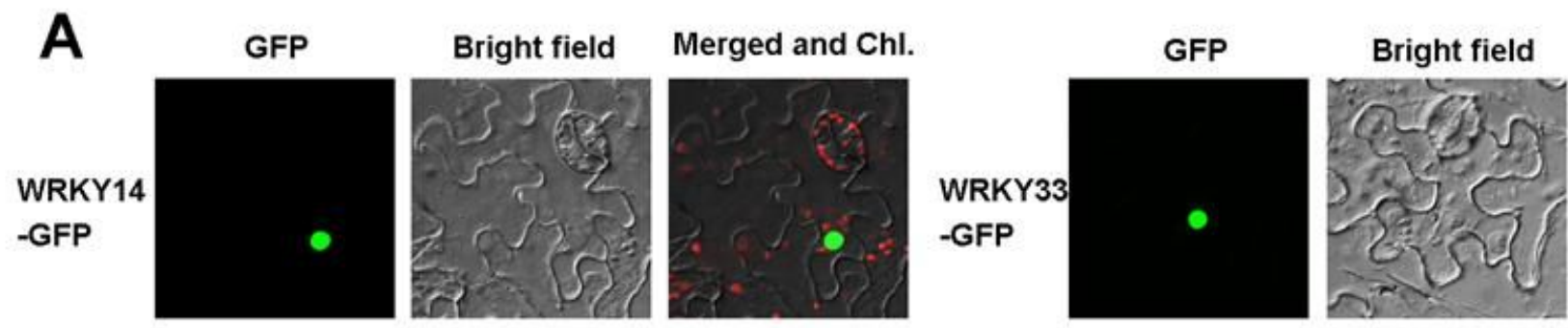

Merged and Chl.
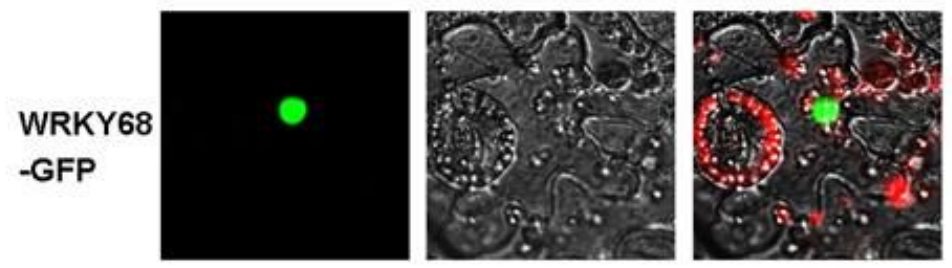

WRKY78

-GFP
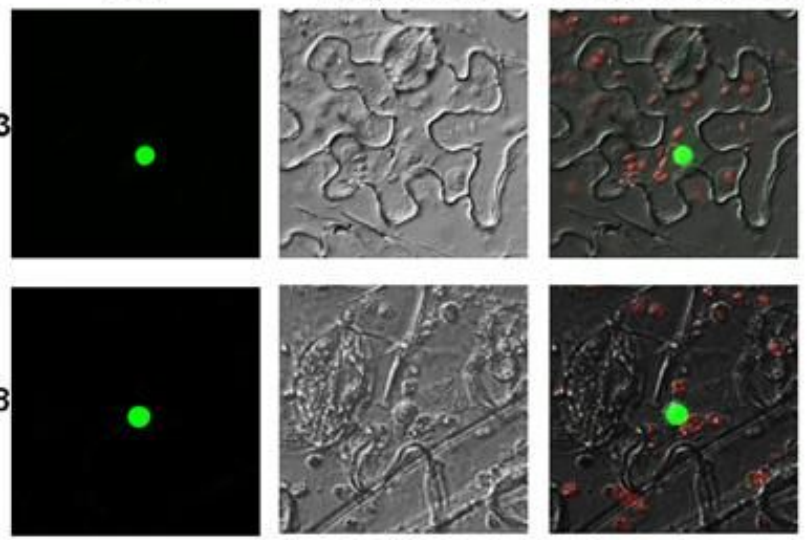

Figure 2

The sub-localization prediction and analysis of SIWRKYs The upper part showed the prediction results of some chloroplast-located SIWRKYs. The bottom showed the nuclear location of SIWRKY14, SIWRKY33, SIWRKY68, SIWRKY78 in Nicotiana benthamiana. Green fluorescence was produced by 35S drived SIWRKY14-GFP, SIWRKY33-GFP, SIWRKY68-GFP, SIWRKY78-GFP. 


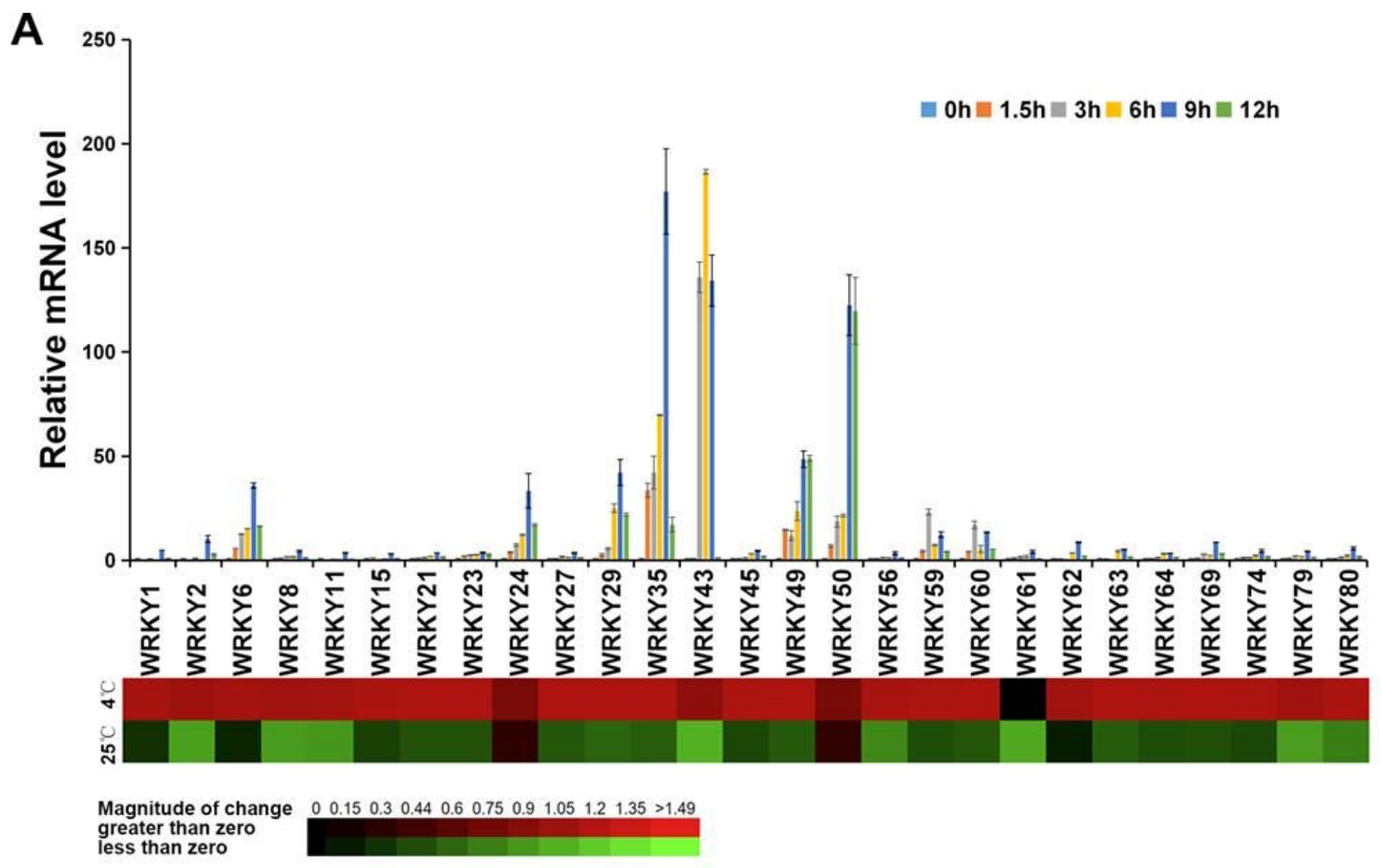

Figure 3

The expression of some SIWRKYs under chilling treatment in tomato The upper part showed the detection of qRT-PCR. All these expression was normalized to ACTIN. Error bars represent the SD of three independent replicates. The bottom part showed the corresponding RNA-seq data in Zhuang et al., 2019. 


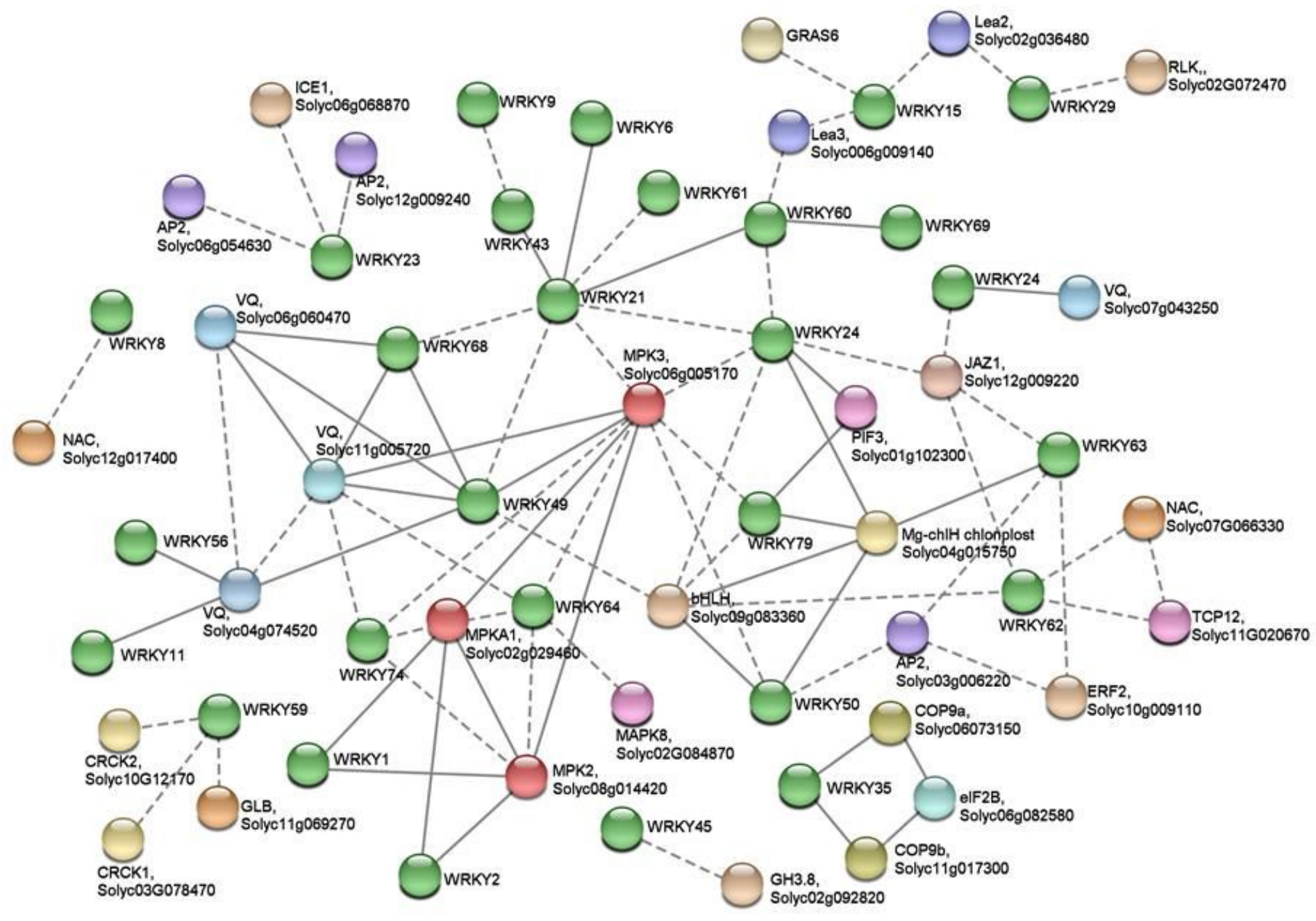

\section{Figure 4}

The interaction network of 27 chilling response SIWRKY proteins The interaction networks of SIWRKY proteins in tomato. Solid line represent confirmed interaction of their corresponding homology proteins in Arabidopsis, dashed line represent unproven protein-protein associations. The green ball indicated SIWRKYs.

\section{Supplementary Files}

This is a list of supplementary files associated with this preprint. Click to download.

- Table.S3.doc

- Table.S2.docx

- Table.S1.doc 